\title{
Time of onset of vardenafil orodispersible tablet in a real-life setting - looking beyond randomized clinical trials
}

\section{Paolo Capogrosso, Eugenio Ventimiglia, Luca Boeri, Alessandro Serino, Andrea Russo, Giovanni La Croce, Umberto Capitanio, Federico Dehò, Francesco Montorsi \& Andrea Salonia}

To cite this article: Paolo Capogrosso, Eugenio Ventimiglia, Luca Boeri, Alessandro Serino, Andrea Russo, Giovanni La Croce, Umberto Capitanio, Federico Dehò, Francesco Montorsi \& Andrea Salonia (2017): Time of onset of vardenafil orodispersible tablet in a real-life setting - looking beyond randomized clinical trials, Expert Review of Clinical Pharmacology, DOI: 10.1080/17512433.2017.1288567

To link to this article: http://dx.doi.org/10.1080/17512433.2017.1288567

Accepted author version posted online: 27 Jan 2017.

Submit your article to this journal ¿

Q View related articles $\sqsubset$

View Crossmark data $־$ 
Publisher: Taylor \& Francis

Journal: Expert Review of Clinical Pharmacology

DOI: $10.1080 / 17512433.2017 .1288567$

Original Research

Time of onset of vardenafil orodispersible tablet in a real-life setting looking beyond randomized clinical trials

Paolo Capogrosso, ${ }^{1,2}$ Eugenio Ventimiglia, ${ }^{1,2}$ Luca Boeri, ${ }^{1}$ Alessandro Serino, Andrea Russo, $^{1,2}$ Giovanni La Croce, ${ }^{1,2}$ Umberto Capitanio ${ }^{1}$, Federico Dehò ${ }^{1}$, Francesco Montorsi, ${ }^{1,2}$ Andrea Salonia ${ }^{1,2}$

${ }^{1}$ Division of Experimental Oncology/Unit of Urology; URI; IRCCS Ospedale San Raffaele, Milan, Italy

${ }^{2}$ Università Vita-Salute San Raffaele, Milan, Italy

Corresponding Author:

Andrea Salonia

Università Vita-Salute San Raffaele

Division of Experimental Oncology/Unit of Urology, URI-Urological Research Institute

IRCCS Ospedale San Raffaele

Via Olgettina 60, 20132 Milan, Italy

Tel. +3902 26437286; Fax +3902 26432969

Email: salonia.andrea@hsr.it 


\section{ABSTRACT}

Background: A rapid onset of action for phosphodiesterase type 5 inhibitors (PDE5is) emerged to be of clinical importance in men treated for erectile dysfunction (ED). Data from randomized clinical trials (RCTs) showed a rapid onset of action for vardenafil 10mg orodispersible tablet (ODT). However, the effectiveness of vardenafil ODT has never been tested in a real-life setting. We assessed the efficacy and time to onset of action of vardenafil ODT in men seeking medical help for ED in the everyday real-life clinical practice.

Research design and methodes: Patients completed a baseline and follow-up International Index of Erectile Function (IIEF), along with a 8-item self-administered questionnaire about onset of action and overall treatment outcomes. Descriptive statistics tested efficacy rates, patient timing of drug intake and time to post-dosing onset of action.

Results: Overall, 118(59.9\%) patients used vardenafil ODT. Satisfactory erections for vaginal penetration were reported in $39(34.5 \%)$ and $26(21.8 \%)$, patients in $\leq 15$ and $\leq 30$, minutes postdosing, respectively. Minimal Clinically Important Differences (MCIDs) criteria and Yang's criteria for responders were obtained in $80(67.8 \%)$ and $72(60.8 \%)$ patients.

Conclusions: This study showed that one in three patients had satisfactory erection for vaginal penetration in less than 15 minutes post-dosing in the real-life setting.

KEYWORDS: erectile dysfunction; phophodiesterase type 5 inhibitors; time to onset; vardenafil; 


\section{INTRODUCTION}

Published data from the past 20 years [1-2], have clearly outlined the importance of personally tailoring both the diagnostic approach and the subsequent therapy for individual patients with erectile dysfunction (ED) [3]. This is especially true as the current real-life setting presents a broad spectrum of ED patients with different age intervals [4,5], comorbidities [6-7] and psychosexological behaviour [8], which translate into different treatment preferences both among physicians and patients themselves $[9,10]$. Tailoring a patient's therapy is achieved through an adequate and commensurate therapeutic approach from the medical and pharmaceutical stand points [11].

In this context, the currently available oral phosphodiesterase type 5 inhibitors (PDE5is) showing different medication features with similar results in terms of efficacy and tolerability $[* * 12,13]$ allow patients to "prefer" a drug also on the basis of specific drug characteristics, thus including the onset of action, the duration of effectiveness and a different route of administration [13,14].

Lack of efficacy has been shown to have a major responsibility in terms of PDE5is drop-off rates, which have been reported to be as high as 50\% per year [15]. Moreover, the rapid onset of action of a PDE5i has been obseryed to have a significant impact in terms of patient's compliance toward a specific drug, either per se or as a perception of lack of efficacy of the compound itself in case of delayed onset of action $[15,16]$. In this context, the orodispersible tablet (ODT) formulation of vardenafil 10mg was launched on the market and was clinically available over the last five years. Data coming from a phase III placebo controlled trial, show a rapid onset of action of vardenafil ODT with almost two-thirds of successful attempts reported within 16-30 minutes post-dosing $\left[17^{*}\right]$.

Beside the available data regarding vardenafil ODT efficacy and tolerability coming from a number of randomized clinical trials (RCTs) $\left[18,{ }^{*} 19\right]$, to the best of our knowledge there are no real-life data from the everyday clinical practice regarding the timing and habits of vardenafil ODT use, the 
actual post-dosing time to onset of action, and its efficacy profile. Such naturalistic data would certainly improve patient-treatment tailoring. Indeed, it is argued that because of the statistical need of limiting confounding factors the highly selected population of RCTs only partially represent the real-life population [20]. In this direction, we sought to evaluate (i) patient behavior regarding timing of drug intake, (ii) time to onset of action post-dosing, and, (iii) the actual effectiveness profile in a cohort of sexually-active, Caucasian-European heterosexual men who had been advised to take vardenafil ODT in the everyday clinical practice at a single academic institution. 


\section{PATIENTS AND METHODS}

\subsection{Population}

The analyses were based on a cohort of 197 consecutive Caucasian-European sexually-active heterosexual patients aged $18+$ who were highly motivated to obtain treatment for ED and who had been advised to take vardenafil ODT between January 2013 and August 2014 at a single academic outpatient clinic. To this aim, ED was defined as the persistent inability to achieve or maintain an erection sufficient for satisfactory sexual performance [20]. Patients were comprehensively assessed with a detailed medical and sexual history, including sociodemographic data. Health-significant comorbidities were scored with the Charlson Comorbidity Index (CCI) [21] both as a continuous or a categorized variable (ie, 0 vs 1 vs $\geq 2$ ). We used the International Classification of Diseases, $9^{\text {th }}$ revision, Clinical Modification (ICD-9-CM). Measured body mass index (BMI), was considered for each patient. For BMI, we used the cut-offs proposed by the National Institutes of Health. Patients were then stratified according to their relationship status (defined as "stable sexual relationship" if the patients had the same partner for 6 or more consecutive months; otherwise "no stable relationship" or widowhood) Patients were requested to complete the International Index of Erectile Function (IIEF) at baseline and at a 3-mo assessment. We used the IIEF-erectile function domain classification as proposed by Cappelleri et al. [22].

Patients were suggested to use vardenafil ODT according to their attitude towards a short versus a long-acting drug. Likewise, we actually considered only patients naïve for vardenafil. Conversely, over the enrollment phase we completely excluded patients either non-responders to previous PDE5is or not suitable for oral treatments and for instance deserving a $2^{\text {nd }}$-line treatment. Patients were thus advised to take supralingually without water, though it could be ingested at the same time as food, depending on patient preference. It was recommended that vardenafil ODT intake occur 1 hour prior to intended sexual intercourse, as previously reported $\left[* 23,{ }^{*} 24\right]$. Patients were instructed to take $10 \mathrm{mg}$ vardenafil ODT on demand, no more than once per day. After a 3-mo course, all 
patients were reassessed in an outpatient setting. At that time, patients were invited to complete a 8item self-administered questionnaire; more precisely, all patients were asked to answer to each question by referring to their most frequent habit over the whole 3-mo course of therapy, with a more precise detail concerning their attitudes throughout the last 4 weeks preceding the follow-up assessment. The survey included sections assessing: i) previous use of any oral compound for ED, as well as effectiveness of the therapy or otherwise; ii) treatment-emergent adverse eyents (TEAEs) (any type), and their eventual continuation even after stopping the drug; iii) actual use of vardenafil ODT; iv) number of sexual attempts using the study medication; v) eventual timing of drug intake before sexual attempt (eg, How long before a sexual attempt did you take the pill?); vi) actual time of post-dosing onset of action (eg, Once taken the pill, how long was it until you achieved an erection rigid enough for satisfactory vaginal penetration?); vii) treatment efficacy (eg, Sexual Encounter Profile question 3 (SEP3: "Did your erection last long enough for you to have successful intercourse?"); and, viii) safety and TEAEs with vardenafil ODT. For the specific purpose of this naturalistic exploratory study, both items concerning the timing of drug intake and the time of postdosing onset of action used a self-reported time estimation. All surveys were self-administered in a clinical setting.

A total of $79(40.1 \%)$ men were excluded because they lacked one or more of the entry criteria: willingness to participate in the survey $(n=45 ; 22.8 \%)$, or the commencement of vardenafil ODT treatment after prescription $(n=34 ; 17.3 \%)$. A sample of 118 patients $(59.9 \%)$ was included in the analysis. Data collection followed the principles outlined in the Declaration of Helsinki; all patients signed an informed consent agreeing to supply their own anonymous information for this and future studies.

\subsection{Main Outcome Measures}

Primary outcome was to assess the actual time to post-dosing onset of action of the study drug. Secondary outcomes were to evaluate the timing of self-medication and to assess any potential clinical predictor of different time-to-onset of action. Likewise, we assessed treatment efficacy 
according to the clinically meaningful improvements as defined by the Minimal Clinically Important Differences (MCIDs) [25] and the Yang's criteria stratification for responders, partial responders and non-responders [26]. Moreover SEP3 rates were calculated overall and per time interval. Finally, treatment compliance and TEAEs were also assessed.

\subsection{Statistical Analysis}

Differences in means and proportions were tested with the paired Student's t-test, and the chisquare $\left(\chi^{2}\right)$ test, respectively. Univariable (UVA) and multivariable (MVA) logistic regression models tested the association between clinical predictors, delayed onset of action (as clinically defined more than 60 minutes after sexual attempt) of vardenafil ODT and non-response to therapy, at 3-mo assessment. Statistical analyses were performed using SPSS statistical software, v 19.0 (IBM Corp., Armonk, NY, USA). All tests were two sided, with a significance level set at 0.05 


\section{RESULTS}

Table 1 shows sociodemographic characteristics for patients who took the study medication $(\mathrm{n}=118$; 59.9\%). Among them, the drug was taken once, $\leq 10$ times, $\leq 20$ times, $21-50$ times, and $>50$ times over the 3-mo survey in $5(4,2 \%) 50(42.4 \%), 20(16.9 \%), 42(35.6 \%)$, and $1(0.8 \%)$ patients, respectively.

Tables $2 \mathrm{a}$ and $2 \mathrm{~b}$ detail timing issues related to vardenafil ODT. Overall, up to $75 \%$ (88) of patients self-reported drug dosing within 1 hour of any type of sexual attempt, as suggested at the time of outpatient evaluation. Conversely, $11(9.3 \%)$ patients reported drug intake $\geq 2$ hours before any sexual attempt (Table 2a). As a whole, 39 (34.5\%) patients reported a rigid erection sufficient for satisfactory vaginal penetration in up to 15 minutes post-dosing, with $70(52.4 \%)$ patients reporting an onset of action within 30 minutes post-dosing (Table 2a). The degree of ED severity was not associated with different timing to onset of action (Table $2 b$ ).

Overall, $33(28.0 \%)$ patients reported at least one TEAE with headache, facial flushing and nasal congestion being reported most frequently in $14(11.8 \%), 11(9 \%)$ and $5(4.3 \%)$ patients, respectively. Overall, TEAES were mostly either mild or mild-to-moderate in severity and did not lead to drug discontinuation in any patient.

Overall, vardenafil ODT promoted a significant improvement in all IIEF domain scores at 3-mo assessment (all $\mathrm{p}=0.0001$; Table 3 ). Moreover, one in two patients reached IIEF-EF domain scores suggestive of normal EF ( $p=0.0001$ ), with satisfaction MCIDs criteria observed in almost $68 \%$ of the cohort.

Yang's criteria for responders were obtained in $72(60.8 \%)$ patients. As a whole, either complete or partial response was achieved in eight out of ten patients according to Yang's criteria for treatment effectiveness (Table 3). The mean per-patient SEP3 success rate were $84.7 \%$ overall (mean No. of attempts 20.3) mean per-patient SEP3 success rates within 15, 30, 60, and more than 60 minutes 
post-dosing reported was $100 \%, 100 \%, 84 \%$ and $46 \%$ with a mean number of attempts of 28.7 , $22.2,19.5$ and 15.1 , respectively.

At logistic regression analysis, no clinical predictors were significantly associated with a delayed onset of action (Table 4). Conversely, high BMI emerged as an independent predictor of being a non-responder to vardenafil ODT according to Yang's criteria (Table 5). 


\section{DISCUSSION}

We assessed real-life data regarding both the timing of drug intake and the post-dosing time of onset of action in a homogenous cohort of sexually-active, heterosexual men treated with on demand vardenafil ODT for a 3-mo course. First, the current findings confirmed previous data $\left[17,{ }^{*} 18,{ }^{*} 23,{ }^{*} 24,27\right]$ suggesting that vardenafil ODT is highly effective at improving erectile function, with a $84.7 \%$ SEP3 success rate, and satisfaction of MCIDs criteria and Yang's criteria for responders in $67.8 \%$ and $60.8 \%$ of the whole cohort, respectively. Patients with higher BMI, emerged to be at higher risk of being a non- responder to drug therapy. Of major clinical interest, $41 \%$ of the patients took the pill only 30 minutes prior to sexual actiyity, with one out of three patients self-reporting a rigid erection sufficient for a satisfactory vaginal penetration within 15 minutes post-dosing, and more than half of the entire cohort reporting a time to onset of action within 30 minutes post-dosing. Of equal clinical significance, at least $10 \%$ of patients voluntarily decided to take the drug more than 2 hours prior to sexual activity. These latter findings highlight the importance of tailoring ED patients according to their specific needs and expectations, as differences in the "sexual ecology" of individual patients, are often clearly present in the real-life setting.

A rapid onset of action of PDE5Is in men seeking medical help for ED is of primary importance to provide patients with adequate confidence to initiate sexual intercourse. To this regard, Axilrod [16] reviewed several studies on PDE5Is showing that time of onset of action is one of the most important attributes for both patients and clinicians, with a consequent potential influences on patient compliance and treatment-adherence. However, naturalistic data regarding the timing and habits related to vardenafil ODT absorption, as well as the actual onset of action in the everyday clinical practice setting, are scarce in the current literature. In this context, Debruyne et al. [17] published the results of a post-hoc integrated analysis performed on data from two 12-week, double-blind, multicenter, randomized, parallel-group, placebo-controlled phase III trials of 
vardenafil ODT. For the specific purposes of their analyses, time intervals (in 15-, 30-, and 60minute increments, up to $\geq 6$ hours after medication intake) were determined for the period between dosing and start of sexual activity. They reported that the mean per-patient SEP3 success rate were $62.5 \%$ for study medication versus $29.4 \%$ for placebo within 15 minutes post-dosing, with corresponding overall SEP3 success rates of $59.8 \%$ and $38.2 \%$, respectively. In the same time span, only $5.3 \%$ and $2.8 \%$ of all sexual activity attempts were initiated by patients taking either vardenafil ODT or placebo, respectively [17]. Our real-life findings showed an even higher overall SEP3 success rate (ie, $84.7 \%$ ), with a $100 \%$ SEP3 success rate for patients self-reporting an onset of action in less than 15 minutes from drug absorption.

The issue of the onset of efficacy of ED drugs has been studied considering the different PDE5Is profiles [28-30]. Padma-Nathan et al. [29], for instance, reported that $35 \%$ and $51 \%$ of sildenafiltreated patients had an erection leading to successful intercourse within 14 and 20 minutes, respectively, post-dosing, with a median time to erection leading to successful intercourse of 36 minutes. Similarly, Shabsigh et al. [30] reported a significant erectogenic response to tadalafil 20 mg from 30 minutes down to 16 minutes after dosing, and from 30 minutes down to 26 minutes after tadalafil $10 \mathrm{mg}$. Of clinical relevance, $52 \%$ of men taking tadalafil $20 \mathrm{mg}$ had at least one successful intercourse attempt within 30 minutes [30]. More recently, data have suggested that avanafil offers a faster onset of action than other PDE5Is when taken in a fasting state [28].

Despite each of these studies investigating the issue of the earliest post-dosing onset of action, they all had considered only either pre-marketing or post-hoc analyses generated on large pre-marketing data, without any accurate investigation in the real-life situation which, in our opinion, has significant clinical importance for better defining the right drug for the right patient at the right time. Real-life studies allow to analyze medical data and provide insight into the real-life effectiveness of a medical intervention [19]; indeed, despite well-known potential biases, assessment of outcomes under eventual "sub-optimal" conditions, give important information on the extent to which an intervention really does what is intended to do in routine circumstances [19]. 
In this context, we reported the efficacy of vardenafil ODT in a real-life cohort, which differs from the highly selected population of RCTs [18*] especially in terms of comorbidity profile and sexual relationship status at the time of the assessment. Moreover, we reported data on the timing of onset of the drug as assessed in a naturalistic setting, thus preserving the spontaneity of the intercourse. The current findings also suggested that vardenafil ODT was well tolerated in our cohort. The rates of incidence and type of TEAEs were comparable with the previously published safety profile $[17, * 23, * 24]$. Finally, due to the real-life setting, we were unable to find any potential predictor of a delayed onset of action of vardenafil ODT, despite including several clinical yariables in the analysis; we believe that potential confounding factors concerning individual metabolic characteristics together with the modality of drug intake could have eventually impacted on the pharmacokinetics profile of the drug [31]. Likewise, we cannot exclude a potential role played by circulating sex hormones, notwithstanding an effect of serum testosterone was described upon the overall efficacy of the PDE5is rather than in regards to the timing of onset per se [32].

Our study is not devoid of limitations. Although this is the first naturalistic study specifically assessing timing issues related to vardenafil ODT intake, this relatively small cohort of men could limit the meaningfulness of our findings. Indeed, the inclusion of only patients referred to a tertiary referral Sexual Medicine outpatient clinic could have substantiated a number of selection biases even in terms of ED seyerity, thus excluding some individuals with either mild ED, who could be less motivated to take a pill, or, on the contrary, some patients with several comorbidities already treated with either high dose PDE5Is or with a $2^{\text {nd }}$-line treatment because of their complexity. However, we believe this methodological flaw could be adequately counterbalanced by the homogeneity of the enrolled sample of men, all of them being sexually-active and highly-motivated to receive an adequate therapy, and therefore potentially more critical of the therapy itself. Moreover, many of these patients were also non-naïve for PDE5Is, thus even more representative of real-life conditions, as more disillusioned and disenchanted with respect to possible treatment failure. As a whole, concerning the assessment of other potential predictors of both efficacy and 
time of onset of vardenafil ODT, for the specific purpose of this study our cohort lacks information regarding the hormonal and metabolic profile, and the recreational habits as well; however, we did not find any association between BMI and the comorbidity profile (as scored using the CCI) which have been demonstrated to severely affect erectile function outcomes [33]. Of clinical relevance, a clear association between circulating sex hormones and the pharmacokinetics of PDE5is had never been detailed. Furthermore, it has been demonstrated that questionnaire-based analyses generally have a lower response rate than other types of investigations, because men tend to be more reluctant to reveal SDs and other impairments in intimate body function when responding to a questionnaire than when speaking directly to their physicians. Second, the application of a non-validated psychometric instrument for assessing drug efficacy in the past three months may have introduced recall bias; indeed, contrary to what is reported in the literature from virtually all studies available to date, we did not use a stopwatch assessment to define the post-dosing timing of onset of action. Though this can certainly have reduced the accuracy and reliability of the results, the goal of our study was to objectify what actually happens in the everyday real-life setting, trying to minimize any potential artifact that could mask or diminish the results themselves. In our opinion, this potential methodological limitation has instead turned into one of the strengths of the study. 


\section{CONCLUSIONS}

This naturalistic study confirmed that on-demand vardenafil ODT is highly effective in men seeking medical help for ED in the real-life setting. Moreover, the current findings offer the first real-life evidence that vardenafil ODT promotes satisfactory erections in less than 30 minutes after dosing in more than one patient out of two, and in more than one third in less than 15 minutes, thus showing even better results compared to RCTs. Safety data closely resembled premarketing results. Overall, these results emphasize the appropriateness of vardenafil ODT as a drug that can be tailored to a patient's real-life needs and expectations. Further studies are needed to extensively investigate and validate the efficacy profile of vardenafil ODT in different sets of patients.

\section{KEY ISSUES}

- Therapy for ED should be properly "tailored" according to patient's characteristics, preferences and expectations

- The time of onset of PDE5is has been shown to have a significant impact in terms of patient's compliance toward a specific drug

- Vardenafil orodispersible tablet (ODT) showed good efficacy and tolerability in previously published RCTs; this manuscript repots novel data regarding the actual post-dosing time to onset of action in the real-life setting

- Our results showed that vardenafil ODT is highly effective in promoting a significant erectile function improvement, with a $67.8 \%$ and a $60.8 \%$ response rate according MCIDs and Yang's criteria, respectively

- Roughly $30 \%$ of patients reported erections sufficient for a satisfactory vaginal penetration within 15 minutes post-dosing, with more than $50 \%$ of them reporting satisfactory erections within 30 minutes post-dosing

- Our results provide the first real-life evidence of an actual short time of onset of vardenafil ODT, along with post-marketing efficacy and safety data, thus supporting the appropriateness of vardenafil ODT as an adequate oral compound tailored adjustable to a patient's real-life needs and expectations 


\section{REFERENCES}

Papers of interest have been highlighted as:

*Papers of interest

** Papers of considerable interest

1. Feldman HA, Goldstein I, Hatzichristou DG, et al. Impotence and its medical and psychosocial correlates: results of the Massachusetts Male Aging Study. J Urol 1994;151:54-61

2. Shabsigh R, Perelman MA, Lockhart DC, et al. Health issues of men: Prevalence and correlates of erectile dysfunction. J Urol 2005; 174:662-7.

3. Mirone V, Fusco F, Rossi A, et al. Tadalafil and vardenafil vs sildenafil: a review of patientpreference studies BJU Int 2009: 103;1212-17

4. Capogrosso P, Colicchia M, Ventimiglia E, et al. One patient out of four with newly diagnosed erectile dysfunction is a young man-worrisome picture from the everyday clinical practice. $\mathrm{J}$ Sex Med 2013;10:1833-41.

5. Nicolosi A, Laumann EO, Glasser DB, et al Global Study of Sexual Attitudes and Behaviors Investigators' Group. Sexual behavior and sexual dysfunctions after age 40: The global study of sexual attitudes and behaviors. Urology 2004; 64:991-7.

6. Scranton RE, Goldstein I, Stecher VJ . Erectile dysfunction diagnosis and treatment as a means to improve medication adherence and optimize comorbidity management. J Sex Med $2013 ; 10: 551-61$.

7. Gandaglia G, Briganti A, Jackson G, et al. A systematic review of the association between erectile dysfunction and cardiovascular disease. Eur Urol 2014;65:968-78.

8. Schmidt HM, Munder T, Gerger H, Frühauf S, Barth J. Combination of psychological intervention and phosphodiesterase-5 inhibitors for erectile dysfunction: a narrative review and meta-analysis. J Sex Med 2014;11:1376-91. 
9. Porst H, Burnett A, Brock G, et al; ISSM Standards Committee for Sexual Medicine. SOP conservative (medical and mechanical) treatment of erectile dysfunction. J Sex Med 2013; $10: 130-71$

10. Pascoal PM, Narciso Ide S, Pereira NM. What is sexual satisfaction? Thematic analysis of lay people's definitions. J Sex Res 2014; 51:22-30.

11. Frederick LR, Cakir OO, Arora H, et al. Undertreatment of erectile dysfunction; claims analysis of 6.2 million patients. J Sex Med 2014;11:2546-53.

12. **Yuan J, Zhang R, Yang Z, et al. Comparative effectiveness and safety of oral phosphodiesterasetype 5 inhibitors for erectile dysfunction: a systematic review and network meta-analysis. Eur Urol 2013; 63:902-12.

In this meta-analysis the authors provide a comprehensive comparison of the efficacy and safety profile of currently available PDE5is.

13. Hatzimouratidis K, Salonia A, Adaikan G et al. Pharmacotherapy for erectile dysfunction: recommendations from the fourth international consultation for sexual medicine (ICSM 2015). J Sex Med 2016; 13:465-88.

14. Hedelin H, Ströberg P Treatment for erectile dysfunction based on patient reported outcomes: to every man the PDE5 inhibitor that he finds superior. Drugs 2005; $65: 2245-51$

15. Corona G, Rastrelli G, Burri A, et al. First-generation phosphodiesterase type 5 inhibitors dropout a comprehensive review and meta-analysis. Andrology 2016 [Epub ahead of print].

16. Axilrod AC. Phosphodiesterase type 5 inhibitor therapy: Identifying and exploring what attributes matter more to clinicians and patients in the management of erectile dysfunction. Curr Med Res Opin 2007; 23:3189-98.

17. Debruyne FM, Gittelman M, Sperling H, et al. Time to onset of action of vardenafil: a retrospective analysis of the pivotal trials for the orodispersible and film-coated tablet formulations. J Sex Med 2011;8:2912-23. 
18. * Martin-Morales A, Gutiérrez-Hernández P, Romero-Otero J, et al; Vadeopen Study Group. Duration of erection: does it really matter? A randomized, double-blind clinical trial to assess the impact of vardenafil ODT on duration of erection and its correlation with patients' and partners' sexual quality of life and duration of intercourse: the VADEOPEN study. J Sex Med $2014 ; 11: 1527-38$

This paper is the most recently published RCT specifically assessing the outcomes of the vardenafil ODT formulation.

19. Saturni S, Bellini F, Braido F, et al. Randomized controlled trials and real life studies. Approaches and methodologies: a clinical point of view. Pulmonary Pharmacoloy \& Threpeutics 2014; 27:129-38

20. NIH Consensus Development Panel on Impotence. JAMA 1993;270:83-90.

21. Charlson ME, Pompei P, Ales KL, et al. A new method of classifying prognostic comorbidity in longitudinal studies: development and validation. J Chronic Dis 1987; 40:373-83.

22. Cappelleri JC, Rosen RC, Smith MD, et al. Diagnostic evaluation of the erectile function domain of the International Index of Erectile Function. Urology 1999; 54:346-51.

23. * Gittelman M, McMahon CG, Rodríguez-Rivera JA, et al. The POTENT II randomised trial: Efficacy and safety of an orodispersible vardenafil formulation for the treatment of erectile dysfunction. Int J Clin Pract 2010; 64:594-603.

Second part of the POTENT trial assessing the efficacy and safety of the orodispersible vardenafil formulation.

24. * Sperling H, Debruyne F, Boermans A, et al. The POTENT I randomized trial: Efficacy and safety of an orodispersible vardenafil formulation for the treatment of erectile dysfunction. $\mathrm{J}$ Sex Med 2010; 7:1497-1507.

First published results of the POTENT trial assessing the efficacy and safety of the orodispersible vardenafil formulation. 
25. Rosen RC, Allen KR, Ni X, et al. Minimal clinically important differences in the erectile function domain of the International Index of Erectile Function scale. Eur Urol 2011;60:101016.

26. Yang M, Ni X, Sontag A, et al. Nonresponders, partial responders, and complete responders to PDE5 inhibitors therapy according to IIEF criteria: validation of an anchor-based treatment responder classification. J Sex Med 2013; 10:3029-37

27. Heinig R, Weimann B, Dietrich H, et al. Pharmacokinetics of a new orodispersible tablet formulation of vardenafil: results of three clinical trials. Clin Drug Investig 2011;31:27-41.

This paper summarized the results of three RCTs assessing the outcomes and pharmacokinetics characteristics of vardenafil ODT.

28. Limin M, Johnsen N, Hellstrom WJ. Avanafil, a new rapid -onset phosphodiesterase 5 inhibitor for the treatment of erectile dysfunction. Expert Opin Investig Drugs 2010; 19:142737.

29. Padma-Nathan H, Stecher VJ, Sweeney M, et al. Minimal time to successful intercourse after sildenafil citrate: results of a randomized, double-blind, placebo-controlled trial. Urology 2003;62:400-03.

30. Shabsigh R, Seftel AD, Rosen RC, et al. Review of time of onset and duration of clinical efficacy of phosphodiesterase type 5 inhibitors in treatment of erectile dysfunction. Urology 2006; 68:689-96.

31. Sanford M. Vardenafil orodispersible tablet. Drugs 2012: 72; 87-98 Eur Urol; 63:902-12.

32. Corona G, Isidori AM, Buvat J, et al. Testosterone supplementation and sexual function: a meta-analysis study. J Sex Med. 2014 Jun;11(6):1577-92.

33. Salonia A, Castagna G, Sacca A, et al. Is erectile dysfunction a reliable proxy of general male health status? The case for the International Index of Erectile Function-Erectile Function domain. J Sex Med 2012; 9:2708-15. 


\section{Funding}

This paper was not funded.

\section{Declaration of Interest}

The authors have no relevant affiliations or financial involvement with any organization or entity with a financial interest in or financial conflict with the subject matter or materials discussed in the manuscript. This includes employment, consultancies, honoraria, stock ownership or options, expert testimony, grants or patents received or pending, or royalties.

\section{Acknowledgements}

The authors thank Roberta Scano Eng for data managing and Dana Kuefner PhD and Chiara Molteni $\mathrm{PhD}$ for reviewing the language in this manuscript.

\section{TABLES}

\section{Table 1}

Descriptive statistics of the cohort of patients participating in the 3-mo study (No. $=118$ )

Patients taking vardenafil ODT

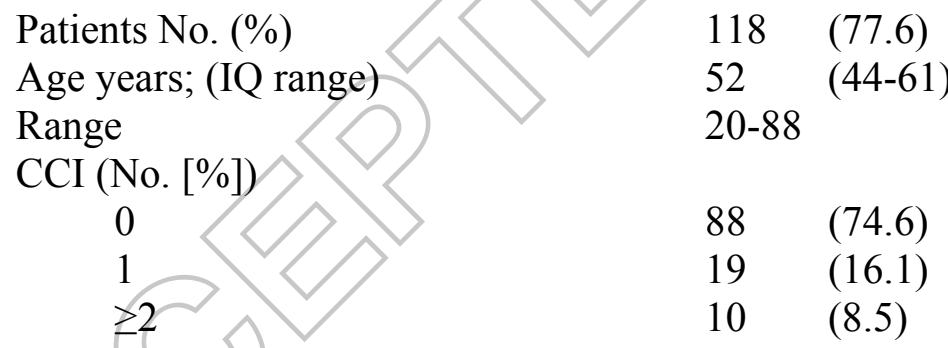

Comorbidities (No. [\%])

Diabetes Type $2 \quad 6 \quad(5)$

Hypertension $45 \quad(37.8)$

Coronary artery disease $\quad 1 \quad(0.9)$

Dyslipidemia 9 (7.6)

Radical prostatectomy for $\mathrm{PCa} \quad 4$

Neurological disease $\quad 7 \quad$ (5.9)

Chronic Kidney disease $\quad 1 \quad(0.9)$

BMI kg/m2; mean (SD) $26.1 \quad$ (4.0)

BMI NIH classification No. (\%)
18.5
$1 \quad(0.9)$ 
$18.5-24.9$

25-29.9

46

(40.7)

$\geq 30$

18

(15.9)

Relationship status No. (\%)

Stable sexual relationship

$112 \quad(95.7)$

No stable sexual relationship

$5 \quad(4.3)$

Educational status No. (\%)

Elementary school

Secondary school

$1 \quad(0.8)$

High school

$23 \quad(19.5)$

University degree

$50 \quad(42.4)$

$44 \quad(37.3)$

Patients naïve for PDE5Is No. (\%) $46 \quad$ (39.0)

Keys: ODT - orodispersable; $\mathrm{SD}=$ standard deviation; $\mathrm{CCI}=$ Charlson Comorbidity Index; $\mathrm{BMI}=$ body mass index; NIH = National Institutes of Health 
Table 2a

Timing issues in terms of vardenafil ODT absorption before sexual attempts

\section{Item 1}

Dosing time before (minutes) any type of sexual attempt

\begin{tabular}{lcll} 
Timing & No. of patients $(\%)$ & Timing & No. of patients $(\%)$ \\
\hline$\leq 30$ & $48(40.7)$ & $\leq 15$ & $39(34.5)$ \\
$30-60$ & $40(33.9)$ & $16-30$ & $26(21.8)$ \\
$60-120$ & $19(16.1)$ & $31-60$ & $27(21.9)$ \\
$120+$ & $11(9.3)$ & $61-120$ & $26(21.8)$
\end{tabular}

Table 2b

Timing to onset of action (minutes) according to IIEF-EF severity at baseline No. of patients (\%)

\begin{tabular}{lcccc}
\hline & $\leq 15$ & $16-30$ & $30-60$ & $61-120$ \\
Mild ED & $8(20.5)$ & $8(33,1)$ & $11(39.2)$ & $5(19.2)$ \\
Mild to Moderate ED & $6(15.4)$ & $9(33.5)$ & $7(25.0)$ & $5(19.2)$ \\
Moderate ED & $19(48.7)$ & $5(16.9)$ & $5(17.8)$ & $12(46.1)$ \\
Severe ED & $6(15.4)$ & $4(16.5)$ & $5(17.8)$ & $4(15.4)$ \\
\hline
\end{tabular}

$\mathrm{P}$-value $=0.11\left(\chi^{2}=14.17\right)$

Keys: $\mathrm{ED}=$ Erectile dysfunction

\section{Item 2}

Timing to onset of action (minutes) 


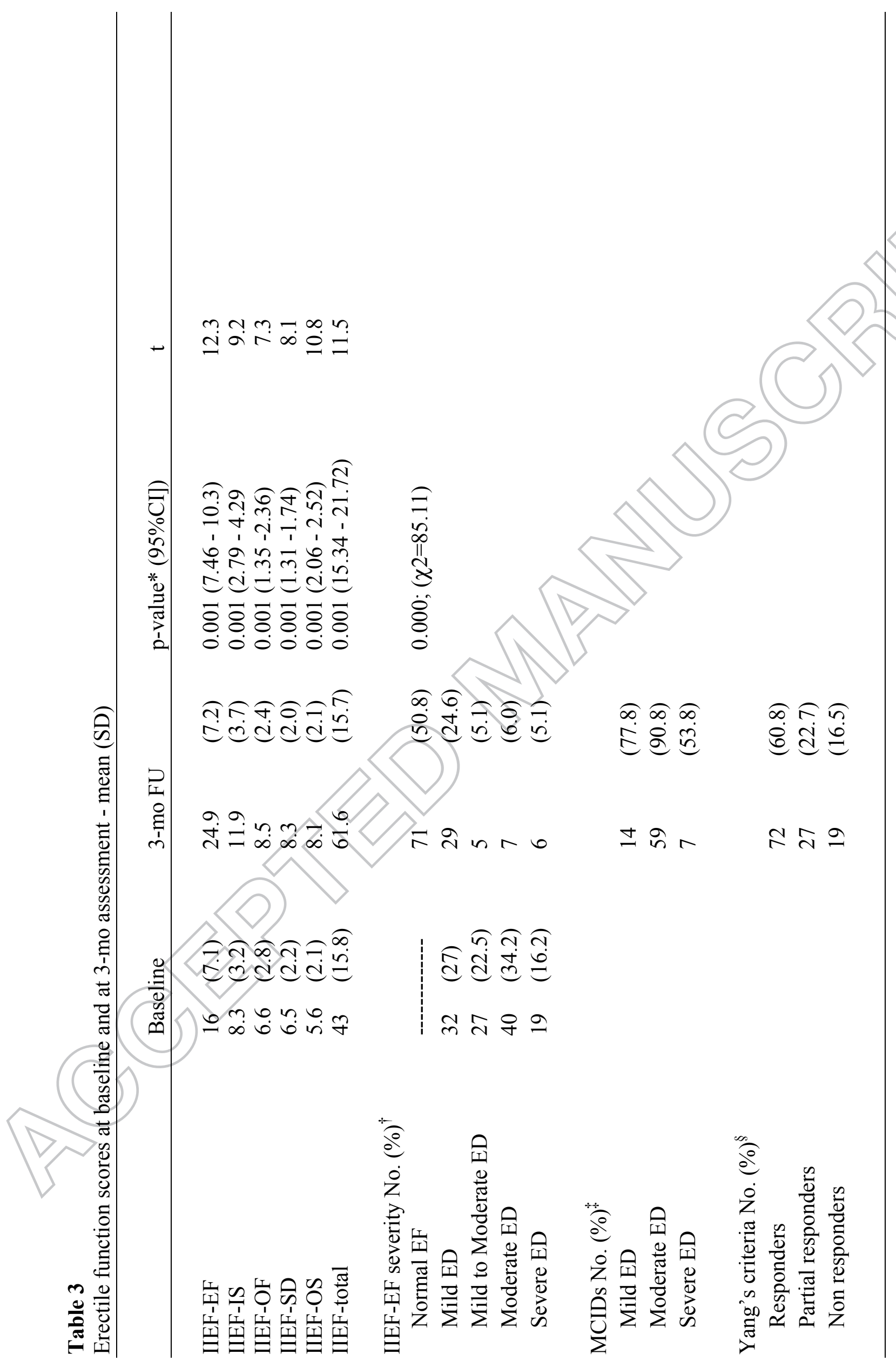




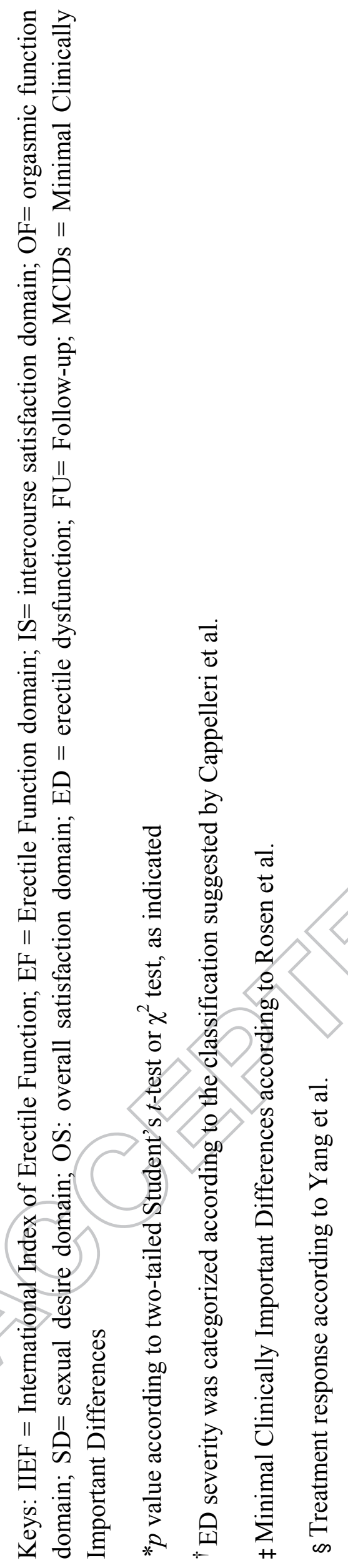




\section{Table 4}

Logistic regression models predicting delayed onset of action post-dosing - OR; $(95 \% \mathrm{CI})$

UVA model

Age

BMI

CCI categorized $(0$ vs $\geq 1)$

PDE5Is naïve vs. non naïve

Baseline IIEF-EF categorized

Mild ED

Mild to Moderate ED

Moderate ED

Severe ED
$1.01 ;(0.97-1.05)$

$1.12 ;(0.99-1.27)$

$2.07 ;(0.73-5.9)$

$0.88 ;(0.34-2.29)$

$0.73 ;(0.09-6.04)$

$1.37 ;(0.22-8.29)$

2.04; $(0.38-10.84)$

$2.06 ;(0.27-15.35)$
MVA model

$0.99 ;(0.95-1.04)$

$1.14 ;(0.99-1.30)$

$2.14 ;(0.65-6.99)$

$0.82 ;(0.29-2.36)$
$0.98 ;(0.11-9.23)$

$1.88 ;(0.27-12.82)$

$2.93 ;(0.48-17.93)$

$2.87 ;(0.34-23.67)$ 


\section{Table 5}

Logistic regression models predicting non-responders according to Yang's criteria - OR; 95\%CI)

UVA model

$1.03 ;(0.98-1.08)$

$1.18 ;(1.01-1.36)$

$2.87 ;(0.89-9.20)$

$2.17 ;(0.64-7.31)$

PDE5Is naïve vs. non naïve

Baseline IIEF-EF categorized

Mild ED

Mild to Moderate ED

Moderate ED

Severe ED

$$
\begin{aligned}
& 0.30 ;(0.04-1.88) \\
& 0.38 ;(0.08-1.77) \\
& 2.62 ;(0.56-12.17) \\
& 0.28 ;(0.37-15.55)
\end{aligned}
$$

1.02; (0.96 - 1.08)

$1.20 ;(1.01-1.43)$

$2.63 ;(0.59-11.63)$

$1.70 ;(0.42-6.38)$

$0.37 ;(0.05-2.68)$

$0.35 ;(0.06-1.94)$

$3.35 ;(0.59-19.01)$

$2.84 ;(0.44-21.67)$

Keys: $\mathrm{BMI}=$ body mass index; $\mathrm{CCI}=$ Charlson Comorbidity Index; PDE5Is $=$ phosphodiesterase type 5 inhibitors; IIEF = International Index of Erectile Function; EF = Erectile Function domain 